\title{
Effects of acute levodopa challenge on resting cerebral blood flow in Parkinson's Disease patients assessed using pseudo- continuous arterial spin labeling
}

Yufen Chen, Peter Pressman, Tanya Simuni, Todd B Parrish, Darren R Gitelman

Introduction Levodopa is the gold-standard for treatment of Parkinson's disease (PD) related motor symptoms. In this study, we used pseudo-continuous arterial spin labeling (pCASL) to quantify changes in cerebral blood flow (CBF) after acute oral administration of levodopa in PD patients. Materials and methods Thirteen patients ( 3 females, age 66.2 \pm 8.7 years) with moderately advanced PD (Hoehn and Yahr stage $>2$ (median 2.5), disease duration $>3$ years) were scanned on a 3T Siemens MR scanner before and after oral levodopa administration. Statistical parametric mapping was used to detect druginduced changes in CBF and its correlation to clinical severity scales. Images were normalized and flipped in order to examine effects on the more affected (left) and less affected (right) cerebral hemispheres across the cohort. Results Levodopa did not change global CBF but increased regional CBF in dorsal midbrain, precuneus/cuneus, more affected inferior frontal pars opercularis and triangularis, bilateral pre- and postcentral gyri, more affected inferior parietal areas, as well as less affected putamen/globus pallidus by $27-74 \%$ ( $p<0.05$, FWE corrected for multiple comparisons). CBF change was negatively correlated with improvement in bradykinesia UPDRS-III subscore in the more affected precentral gyrus, and total predrug UPDRS-III score in the mid-cingulate region. Druginduced CBF change in a widespread network of regions including parietal and postcentral areas was also negatively correlated with the predrug rigidity UPDRS-III subscore.

Conclusion These findings are in line with prior reports of abnormal activity in the nigrostriatal pathway of PD patients and demonstrate the feasibility of pCASL as a neuroimaging tool for investigating in vivo physiological effects of acute drug administration in PD. 
1 Effects of acute levodopa challenge on resting cerebral blood flow in Parkinson's Disease

2 patients assessed using pseudo-continuous arterial spin labeling

3 Yu Fen Chen ${ }^{\mathrm{a}, *}$, Peter Pressman ${ }^{\mathrm{b}}$, Tanya Simuni ${ }^{\mathrm{c}}$, Todd B. Parrish ${ }^{\mathrm{a}}$ and Darren Gitelman ${ }^{\mathrm{c}, \mathrm{d}, \mathrm{e}}$

4

5 aDepartment of Radiology, Feinberg School of Medicine, Northwestern University, Chicago, IL 6 USA

7 bDepartment of Neurology, Memory and Aging Center, University of California, San Francisco, 8 CA USA

9 'Department of Neurology, Feinberg School of Medicine, Northwestern University, Chicago, IL 10 USA

11 dAdvocate Lutheran General Hospital, Park Ridge, IL USA

12 eDepartment of Medicine, Chicago Medical School at the Rosalind Franklin University of

13 Medicine and Science, North Chicago, IL USA 


\section{Word count}

Abstract: 263

Article: 4101

References: 54

Tables: 3

Figures: 3

\section{Abbreviations}

$\mathrm{PD}=$ Parkinson's disease; FDG-PET= Fluorodeoxyglucose positron emission tomography; $\mathrm{PDRP}=\mathrm{PD}$ related pattern; $\mathrm{CBF}=$ cerebral blood flow; $\mathrm{BOLD}-\mathrm{fMRI}=$ blood oxygenation level dependent functional MRI; SMA= supplementary motor area; $\mathrm{ASL}=$ arterial spin labeling; UPDRS=Unified Parkinson's Disease Rating Scale; LDE=levodopa equivalent; $\mathrm{pCASL}=$ pseudo-continuous arterial spin labeling; $\mathrm{SPM}=$ statistical parametric mapping; $\mathrm{SNR}=$ signal to noise ratio; $\mathrm{CMRO}_{2}=$ cerebral metabolic rate of oxygen 
39

40

41

42

43

44

45

\section{Abstract}

Introduction Levodopa is the gold-standard for treatment of Parkinson's disease (PD) related motor symptoms. In this study, we used pseudo-continuous arterial spin labeling (pCASL) to quantify changes in cerebral blood flow (CBF) after acute oral administration of levodopa in PD patients.

Materials and methods Thirteen patients (3 females, age $66.2 \pm 8.7$ years) with moderately advanced PD (Hoehn and Yahr stage $>2$ (median 2.5), disease duration $>3$ years) were scanned on a 3T Siemens MR scanner before and after oral levodopa administration. Statistical parametric mapping was used to detect drug-induced changes in $\mathrm{CBF}$ and its correlation to clinical severity scales. Images were normalized and flipped in order to examine effects on the more affected (left) and less affected (right) cerebral hemispheres across the cohort.

Results Levodopa did not change global CBF but increased regional CBF in dorsal midbrain, precuneus/cuneus, more affected inferior frontal pars opercularis and triangularis, bilateral preand postcentral gyri, more affected inferior parietal areas, as well as less affected putamen/globus pallidus by $27-74 \%$ ( $\mathrm{p}<0.05$, FWE corrected for multiple comparisons). CBF change was negatively correlated with improvement in bradykinesia UPDRS-III subscore in the more affected precentral gyrus, and total predrug UPDRS-III score in the mid-cingulate region. Drug-induced CBF change in a widespread network of regions including parietal and postcentral areas was also negatively correlated with the predrug rigidity UPDRS-III subscore.

Conclusion These findings are in line with prior reports of abnormal activity in the nigrostriatal pathway of PD patients and demonstrate the feasibility of pCASL as a neuroimaging tool for investigating in vivo physiological effects of acute drug administration in PD. 
61

62 Key words: arterial spin labeling, cerebral blood flow, Parkinson's disease, levodopa,

63 pharmacological MRI 
64

\section{Introduction}

Parkinson's disease (PD) is a neurodegenerative disorder where the classical pathophysiology is the loss of dopaminergic neurons in the substantia nigra and disruption of the nigrostriatal pathway(Stoess1 2012), which results in motor dysfunction. Levodopa, a dopamineprecursor, is currently one of the most effective treatments for $\mathrm{PD}$ (Hornykiewicz 2010). While the mechanism of action of levodopa is due to its ability to at least partially replete dopamine deficiency, few studies to-date have focused on studying the effects of levodopa on brain physiology. Due to the lack of validated biomarkers for PD diagnosis or disease progression, current drug development in PD uses clinical outcome scales as measures of efficacy. This precludes quick assessment of new drugs in humans. Development of imaging biomarkers to assist in assessment of drug mechanism and target validation is crucial for development of effective new treatment regimens.

Neuroimaging techniques have been invaluable in providing the means to study drug effects in-vivo, noninvasively. Fluorodeoxyglucose positron emission tomography (FDG-PET) has revealed in PD patients a network of brain regions with reduced glucose metabolism known as the PD Related Pattern (PDRP)(Eidelberg 2009). The strength of its expression correlates with symptom severity, and is also reduced by levodopa treatment(Eidelberg 2009). Similarly, ${ }^{15} \mathrm{O}-$ PET has also shown that levodopa increases both resting cerebral blood flow (CBF)(Kobari et al. 1995) and motor activity(Feigin et al. 2002) in the putamen and thalamus of PD patients compared to healthy controls. Such findings have also been corroborated in blood oxygenation level dependent functional MRI (BOLD-fMRI) studies, which found that levodopa normalizes PD-related hypoactivity in regions such as putamen, M1 and supplementary motor area (SMA)(Buhmann et al. 2003; Tang \& Eidelberg 2010). Despite the high signal-to-noise ratio and 
87 ease of implementation, traditional BOLD techniques are non-quantitative and dependent on

88 multiple physiological processes including blood flow, blood volume and oxygen consumption,

89

90

91

92

93

94

95

96

97

98

99

100

101

102

103

104

105

106

107

108

and can thus be difficult to interpret in situations where more than one of these processes are affected.

Arterial spin labeling (ASL)(Detre et al. 1992) is another MRI technique that provides a quantitative measure of CBF by magnetically labeling blood spins without the need for contrast agent injection. In this regard, ASL is more similar to ${ }^{15} \mathrm{O}-\mathrm{PET}$ than traditional BOLD fMRI techniques as it provides a quantitative assessment of baseline physiology, albeit without radioactive exposure. Recent ASL studies have revealed similar hypoperfusion patterns in PD patients compared to controls as previously shown with PET and SPECT imaging(Eidelberg 2009; Paschali et al. 2010; Song et al. 2015), including regions such as posterior parieto-occipital cortex, precuneus and cuneus and middle frontal gyri(Fernandez-Seara et al. 2012; Kamagata et al. 2011; Melzer et al. 2011). Furthermore, expression of the FDG-PET PDRP network is similarly increased in ASL CBF maps of PD patients compared to healthy controls and positively correlated to the expression in FDG-PET data of the same cohort(Ma et al. 2009; Teune et al. 2014).Since the ASL signal arises from magnetically labeled blood spins, which have a longitudinal relaxation time of $1.6 \mathrm{~s}$ at $3 \mathrm{~T}$, ASL can be easily repeated during a single scan session, making it well-suited for assessing the effects of a pharmacological challenge on brain activity (see Wang et al. (2006) (Wang et al. 2011) for a review). The quantitative nature of ASL and its excellent reproducibility(Chen et al. 2011; Chen et al. 2010; Gevers et al. 2009; Hermes et al. 2007) also allow it to assess brain activity in the absence of tasks, making it especially attractive for studying patients by eliminating the potential confound of task performance. In this 
109 feasibility study, we aim to determine whether ASL can detect the effects of levodopa on resting

$110 \mathrm{CBF}$ in a cohort of PD patients.

111

112 Methods

113 Subjects

114 A total of 16 patients (3 females, age 66.2 \pm 8.7 years) with moderate PD severity (Hoehn 115 and Yahr stage $>2$ (median 2.5)range, disease duration $>$ mean ( SD ) 3 years) were recruited

116 for the current study. One participant did not complete the postdrug session as the predrug

117 images were corrupted by severe motion artifacts. Two other participants were removed from

118 final analysis due to signal dropouts in the images which resulted in exaggerated CBF changes.

119 Thus the final cohort consisted of 13 subjects. All participants were on a stable dose of

120 carbidopa-levodopa therapy for at least 3 months prior to enrollment in the study. None of the

121 participants had any visible head tremor as assessed by the clinicians on our team (DRG \& PP)

122 after dopaminergic medication was withheld approximately 12 hours. This study was approved

123 by the Northwestern Institutional Review Board (IRB ID: STU00049159) and written informed

124 consent was obtained from all subjects prior to scanning. See Table 1 for a summary of the

125 demographic details. 
Table 1. Demographics, UPDRS-III ratings and Levodopa-equivalence (LDE) in mg.

\section{Demographics $(\mathbf{n}=13)$}

\begin{tabular}{|c|c|c|c|}
\hline \multicolumn{3}{|l|}{ Age, years (mean \pm s.d.) } & $65 \pm 7$ \\
\hline \multicolumn{3}{|l|}{ Female:male } & $3: 10$ \\
\hline \multicolumn{3}{|l|}{ Duration of disease (years) } & $8 \pm 6$ \\
\hline \multicolumn{3}{|l|}{$\mathrm{LDE}^{*}, \operatorname{mg}($ mean \pm s.d.) } & $270 \pm 132$ \\
\hline \multicolumn{3}{|l|}{ L vs R dominant symptoms } & $5: 8$ \\
\hline \multicolumn{4}{|c|}{ Drug Response $(n=13)$} \\
\hline & pre & post & \%improvement \\
\hline UPDRS-III total(mean \pm s.d.) & $19 \pm 6$ & $9 \pm 6$ & $52.0 \pm 28.3$ \\
\hline Bradykinesia subscore $^{\dagger}$ & $7 \pm 4$ & $4 \pm 4$ & $49.1 \pm 38.8$ \\
\hline Tremor subscore & $3 \pm 2$ & $1 \pm 2$ & $58.3 \pm 43.3$ \\
\hline Rigidity subscore & $5 \pm 2$ & $3 \pm 2$ & $46.8 \pm 34.5$ \\
\hline Posture subscore & $3 \pm 1$ & $1 \pm 1$ & $55.1 \pm 39.8$ \\
\hline
\end{tabular}

*LDE $=$ levodopa equivalents based on Tomlinson et al (2010) for the morning dose of dopaminergic medications.

†See text for the formulas for the calculation of the UPDRS subscores.

Study Protocol

Subjects were instructed to refrain from taking levodopa for at least 12 hours prior to arrival at the MRI facility. Upon arrival, subjects underwent an assessment that included the

130 Unified Parkinson's Disease Rating Scale - motor (UPDRS-III) (Fahn S 1987) and then had an initial MR scanning session that consisted of the high resolution anatomical and ASL sequences. This session was designated the OFF or predrug state. Participants were then removed from the scanner and given an oral dose of carbidopa-levodopa approximately equivalent to $125 \%$ of their usual morning dose of dopaminergic medications calculated as levodopa equivalents (LDE)(Tomlinson et al. 2010). After 1 hour, subjects were re-evaluated with the UPDRS-III and then had a second scanning session and ASL acquisition (ON or postdrug state). Although we had initially specified that subjects should show at least a 30\% improvement in their UPDRS-III 
138 motor score for inclusion in the analysis, because of the small number of subjects we ended up

139 retaining the 3 subjects with less than a $30 \%$ improvement $(10 \%, 19 \%$ and $25 \%)$.

140 UPDRS-III subscores were calculated as follows for each session: 1) bradykinesia: sum

141 of scores for finger tapping, hand movements, pronation-supination movements, leg agility and

142 body bradykinesia; 2) rigidity: sum of rigidity scores for upper and lower extremities; 3 ) tremor:

143 sum of rest and action tremor scores for upper and lower extremities; and 4) posture: sum of

144 scores for posture, gait and postural stability. Lateralized scores were summed to generate a

145 single number for each subscore for each subject.

146 All data were collected on a Siemens TIM Trio 3T whole-body scanner (Erlangen,

147 Germany), equipped with a 12-channel receive-only head coil. A high resolution T1-weighted

148 anatomical image was acquired using an MPRAGE sequence (TR/TE 2300/2.98 ms, TI=900 ms,

149 flip angle $=9^{\circ}$, GRAPPA acceleration factor $=2$, matrix $256 \times 256$, voxel size $1 \times 1 \times 1 \mathrm{~mm}^{3}, 176$

150 slices). Cerebral blood flow maps were collected in both scan sessions using pseudo-continuous

151 arterial spin labeling (pCASL) (Dai et al. 2008). ASL images were acquired with gradient-echo

152 echo-planar imaging (EPI) with a field-of-view of $22 \mathrm{~cm}$, and in-plane resolution of $3.5 \times 3.5$

$153 \mathrm{~mm}^{2}$. Twenty, $6 \mathrm{~mm}$ axial slices with a $2 \mathrm{~mm}$ gap were prescribed to cover the entire brain

154 including the cerebellum. Based on previous ASL studies on PD patients, labeling duration and

155 post-labeling delay were both set to 1.5s(Fernandez-Seara et al. 2012; Melzer et al. 2011). Forty

156 pairs of tag and control images were acquired in an interleaved fashion, resulting in a scan time

157 of 5.5 minutes.

\section{Data Processing}

159 All images were processed using SPM8 (Wellcome Trust Centre for Neuroimaging,

160 London, UK) and in-house developed scripts written in Matlab R2010b (Mathworks, Natick, 
161 MA, USA). The raw EPI images from both ASL scans were first co-registered to the high

162 resolution anatomical image collected in session 1 and then motion-corrected by aligning all

163 images to the first image of the session using a rigid-body, six parameter transformation.

164 Pairwise subtraction images were computed for each control and tag pair, and outliers were

165 excluded from the computation of the mean perfusion weighted image based on previously

166 published guidelines(Wang et al. 2008). An image was labeled as outlier if it fit any of the

167 following criteria: a) rotation greater than 0.8 degrees, b) translation greater than $0.8 \mathrm{~mm}$ between

168 the control and tag pair, c) mean signal more than two standard deviations from the mean signal

169 of the time-series or d) mean background noise more than two standard deviations from the mean

170 background noise of the time-series. On average, $7 \pm 3$ and $8 \pm 4$ pairs were labeled as outliers in

171 the pre- and post-drug sessions, respectively, using the above criteria, indicating no significant

172 difference in data quality between sessions. The mean perfusion weighted image was converted

173 to CBF in physiological units using a previously published model(Wang et al. 2008). Mean

174 global gray matter (GM) CBF values for baseline and post-drug conditions were computed from

175 subject-specific masks encompassing voxels containing at least 50\% GM, generated by

176 segmenting the T1 image with the VBM8 toolbox (http://dbm.neuro.uni-jena.de/vbm)(Ashburner

177 \& Friston 2000) and compared using a paired t-test. The low resolution CBF images were then

178 spatially normalized to MNI space by applying the transformation matrices obtained from

179 normalizing the high resolution anatomical images to a symmetrical template using VBM8.

180 To determine the most affected hemisphere across subjects, a motor impairment laterality

181 index was calculated using the formula $(\mathrm{R}-\mathrm{L}) /(\mathrm{R}+\mathrm{L})$, where $\mathrm{R}$ and $\mathrm{L}$ represent the sum of the

182 right and left limb UPDRS-III scores in the OFF drug condition respectively. Four subjects had a

183 laterality index of less than 0 , indicative of left-predominant symptoms, which would affect the 
184 right hemisphere. One subject had a laterality index of 0 , with reported onset of symptoms on the

185 left side. Since prior animal and human studies have demonstrated a close relationship between

186 symptom laterality and asymmetry of biochemical changes in the brain(Eidelberg et al. 1990;

187 Guttman et al. 1988; Huang et al. 2001; Morrish et al. 1995; Prakash et al. 2012; Rinne et al.

188 1993; Yang et al. 2007), and CBF is related to synaptic activity(Lauritzen 2001a) that is also

189 affected by underlying biochemical activity, images from these five subjects were flipped such

190 that the most affected hemisphere for all subjects was on the left side in MNI space, thereby

191 minimizing pathological variability due to symptom laterality. This is similar to the approach

192 employed by another study that designated regions of interest as contra- or ipsilateral depending

193 on the side of symptoms(Schuff et al. 2015). All spatially normalized CBF maps were smoothed

194 with an 8mm full-width at half maximum Gaussian kernel.

195

\section{Statistical comparisons}

Threshold-free cluster enhancement (TFCE)(Smith \& Nichols 2009) and nonparametric permutation testing(Nichols \& Holmes 2002) with 2000 permutations were used for statistical analysis as these techniques have previously been shown to have superior sensitivity than traditional parametric statistics, and are particularly well-suited for studies with low degrees of freedom. Calculations were performed using the TFCE toolbox for SPM8. All results were thresholded at $\mathrm{p}<0.05$, with family-wise error (FWE) correction for multiple comparisons.

\section{Paired t-test: Predrug vs. postdrug CBF}

The predrug and postdrug CBF maps of all subjects were entered into a voxelwise paired ttest to explore the effects of levodopa on CBF. Two separate t-tests were performed on both 
the quantitative $\mathrm{CBF}$ maps and $\mathrm{CBF}$ maps proportionally scaled to 50 in order to minimize variance due to individual differences in CBF. Statistical analysis was limited to voxels with a raw signal to noise ratio (SNR) of at least 1 in both sessions for all 13 subjects, where raw SNR was calculated as the mean signal over time divided by the standard deviation of signal

\section{Regression: drug-induced change in CBF vs. change in UPDRS III}

To explore the relationship between levodopa-induced CBF changes and changes in motor function, normalized CBF difference maps were calculated by subtracting the predrug maps

\section{Regression: drug-induced CBF change vs. predrug UPDRS-III}

\section{Regression: drug-induced CBF changes vs. LDE}

A similar approach was used to determine the relationship between drug-induced normalized $\mathrm{CBF}$ changes and baseline symptom severity by regressing the $\mathrm{CBF}$ difference maps against predrug UPDRS-III total and subscores.

We also examined whether the induced CBF changes were related to the amount of levodopa administered with a multiple regression analysis of the drug-induced normalized CBF change maps using LDE values as the covariate of interest. 


\section{Results}

As listed in table 1, participants had an average $52.0 \pm 28.3 \%$ improvement in their

UPDRS-III total score. The minimum improvement for any subject was $10 \%$. Also noted in

Table 1, the average improvement in subscores was $49.1 \pm 38.8 \%$ for bradykinesia, $46.8 \pm 34.5 \%$

for rigidity, $58.3 \pm 43.3 \%$ for tremor and $55.1 \pm 39.8 \%$ for posture/gait.

\section{Paired t-test: Predrug vs. postdrug CBF}

The mean global CBF extracted from voxels containing at least 50\% GM was $46.2 \pm 7.1$ $\mathrm{ml} / 100 \mathrm{~g} / \mathrm{min}$ before levodopa and $47.6 \pm 7.9 \mathrm{ml} / 100 \mathrm{~g} / \mathrm{min}$ after levodopa, the difference was not statistically significant $\left(\mathrm{t}_{12}=-0.78, \mathrm{p}=0.22\right)$.

In the voxel-wise analysis, no significant CBF changes were detected with the unscaled, raw, quantitative CBF maps, which was as expected since the inter-subject differences in global CBF far outweighed the drug-induced changes. Thus, only results from the proportionally scaled images are reported below. Figure 1 shows the clusters with significant CBF increases after levodopa, thresholded at $\mathrm{p}<0.05$ FWE, overlaid onto a high-resolution anatomical image. The image is oriented so that the "more affected" side of the brain (subject brains flipped as described above) is on the left side of the image. The colorbar represents the range of log scaled p-values. No levodopa-induced CBF decreases were detected. A summary of clusters, including MNI coordinates, FWE p-values, and approximate anatomical localization identified using the automatic anatomic labeling (AAL) toolbox(Tzourio-Mazoyer et al. 2002) for SPM are listed in Table 2. Mean CBF change extracted from a 5mm sphere centered on the peak voxel of each cluster is also shown in Table 2. Levodopa induced CBF increases in 


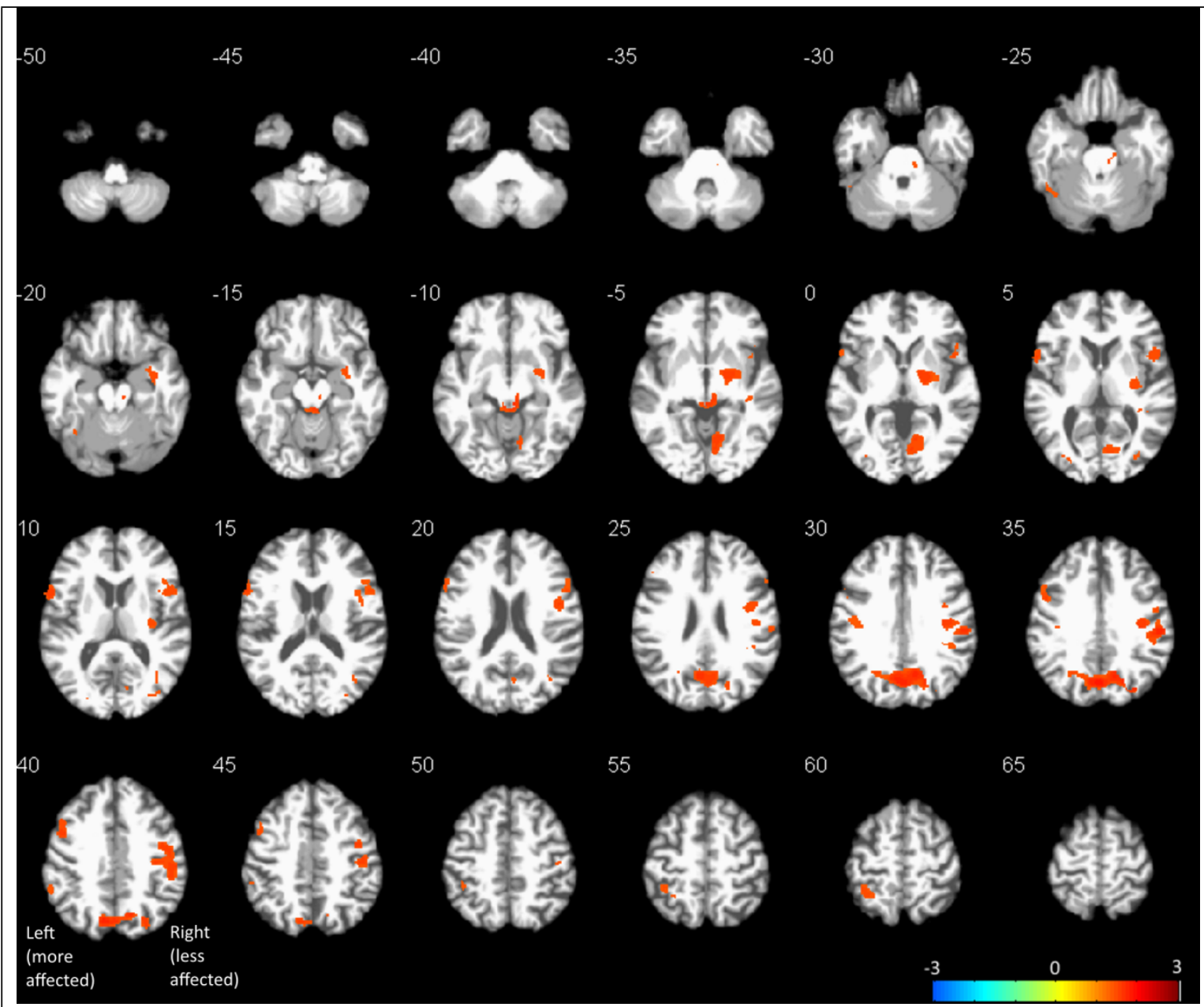

Figure 1. Results of voxel-wise paired t-test between pre- and post-drug CBF maps thresholded at $\mathrm{p}<0.05$, FWE corrected for multiple comparisons, overlaid onto a representative subject's spatially normalized anatomical image. Images are oriented so that the "more affected" side of the brain is on the left side of the image. Orange represents CBF increases. No CBF decreases were detected at the statistical threshold used. 


\begin{tabular}{|c|c|c|c|c|}
\hline $\mathbf{x}, \mathbf{y}, \mathbf{z}, \mathbf{m m}$ & $\begin{array}{c}\text { No. } \\
\text { voxels }\end{array}$ & $\begin{array}{c}\text { FWE } \\
\text { p-value }\end{array}$ & label & $\begin{array}{l}\text { \% CBF } \\
\text { change }\end{array}$ \\
\hline $0-7033$ & 2695 & 0.016 & Cuneus, precuneus & 33.4 \\
\hline $56-2434$ & 3046 & 0.020 & $\begin{array}{c}\text { SupraMarginal (LA), } \\
\text { Frontal_Inf_Oper (LA), } \\
\text { Postcentral (LA), Precentral (LA) }\end{array}$ & 26.9 \\
\hline $4-33-9$ & 544 & 0.027 & midbrain & 73.6 \\
\hline $20-6-3$ & 1150 & 0.029 & Pallidum (LA), putamen (LA) & 44.0 \\
\hline-60119 & 461 & 0.030 & Frontal_Inf_Oper (MA) & 50.6 \\
\hline $10-64-5$ & 863 & 0.030 & Lingual (LA) & 33.0 \\
\hline-48839 & 428 & 0.031 & Precentral (MA) & 31.0 \\
\hline$-34-4660$ & 314 & 0.043 & Parietal_Inf (MA) & 61.1 \\
\hline $34-799$ & 108 & 0.043 & Occipital_Mid (LA) & 40.9 \\
\hline$-40-1830$ & 146 & 0.044 & Postcentral (MA) & 47.1 \\
\hline $38-6915$ & 77 & 0.044 & White matter & 47.8 \\
\hline$-40-57-23$ & 108 & 0.045 & Cerebelum_6(LA) & 36.2 \\
\hline $40-27-6$ & 57 & 0.045 & White matter & 32.1 \\
\hline$-27-857$ & 59 & 0.045 & Occipital_Mid (MA) & 47.3 \\
\hline$-58-4539$ & 77 & 0.047 & Parietal_Inf (MA) & 30.1 \\
\hline$-8-28-14$ & 2 & 0.048 & midbrain & 62.5 \\
\hline $38-396$ & 26 & 0.049 & White matter & 85.8 \\
\hline-503025 & 5 & 0.050 & Frontal Inf Tri (MA) & 41.8 \\
\hline
\end{tabular}
with drug-induced changes in $\mathrm{CBF}$. This cluster, located in the left middle cingulate, is and FWE p-value are shown in Table 3. 


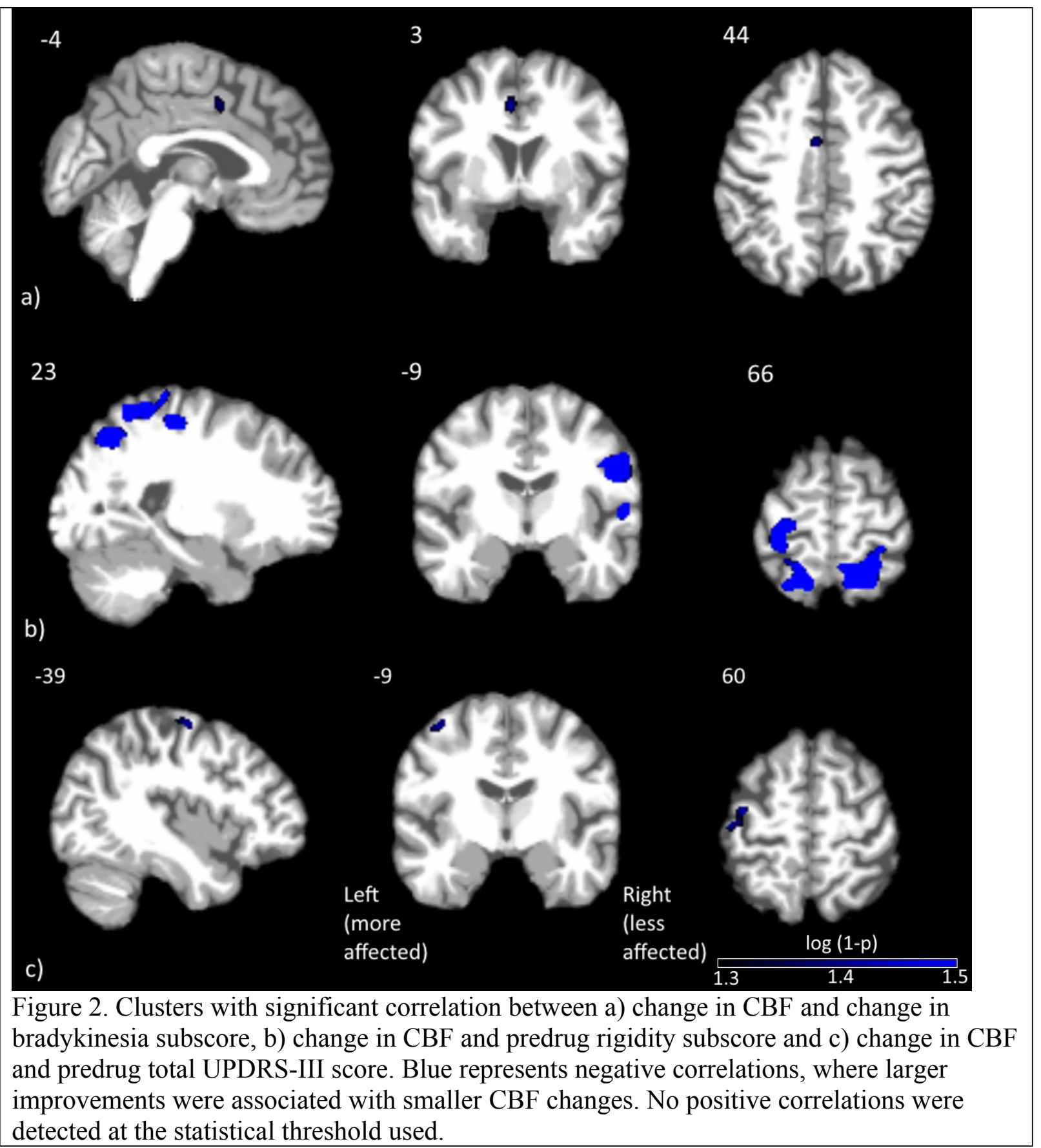

264 3. Regression: drug-induced CBF change vs. predrug UPDRS-III

265 Clusters with significant correlations between change in CBF and predrug UPDRS-III scores are shown in Figure 2b-c. Details on the clusters are summarized in Table 3. To demonstrate 
267 that the results were not driven by any outliers, scatterplots of $\Delta \mathrm{CBF}$, extracted from

268 significant clusters and the corresponding UPDRS scores are shown in Figure 3. Regression

269 lines and adjusted $\mathrm{R}^{2}$ values are also shown on the plots, and are intended to demonstrate the

270 strength of the regression in these clusters, not as an independent analysis of the data (i.e.

271 circular analysis(Kriegeskorte et al. 2010)). The total UPDRS-III and rigidity subscores were

272 significantly correlated with change in CBF. Total UPDRS-III score was negatively correlated to CBF changes in the more affected precentral gyrus. The rigidity subscore was negatively correlated to $\mathrm{CBF}$ changes in multiple areas including bilateral postcentral gyri, supplementary motor area and parietal and temporal regions.

Table 3. List of clusters with significant correlations between normalized $\triangle \mathrm{CBF}$ and predrug or change in UPDRS scores. MNI coordinates of the peak voxel are given in mm, as well as the number of voxels in each cluster and FWE p-value.

\begin{tabular}{ccccc}
\hline & $\mathbf{x , y}, \mathbf{z}, \mathbf{m m}$ & No. voxels & $\begin{array}{c}\text { FWE } \\
\text { p-value }\end{array}$ & label \\
\hline $\begin{array}{c}\text { BuPDRS } \\
\text { (negative) }\end{array}$ & $-4,3,43$ & 88 & 0.043 & Cingulum_Mid (MA) \\
$\begin{array}{c}\text { Pre-drug UPDRS } \\
\text { Rigidity (negative) }\end{array}$ & $38,-57,54$ & 1997 & 0.009 & Angular(LA) \\
& $48,-12,30$ & 987 & 0.010 & Postcentral (LA) \\
& $58,-27,12$ & 4617 & 0.045 & Temporal_Sup (LA) \\
& $-27,-60,61$ & 2547 & 0.019 & Parietal_Sup (MA) \\
& $-46,-48,52$ & 457 & 0.032 & Parietal_Inf (MA) \\
& $2,-21,72$ & 6 & 0.044 & Supp_Motor_Area (LA) \\
& $-34,-24,49$ & 106 & 0.045 & Postcentral (MA) \\
Total (negative) & $-39,-9,60$ & 134 & 0.043 & Precentral (MA) \\
\hline MA=more affected; LA=less affected. & & & \\
\hline
\end{tabular}




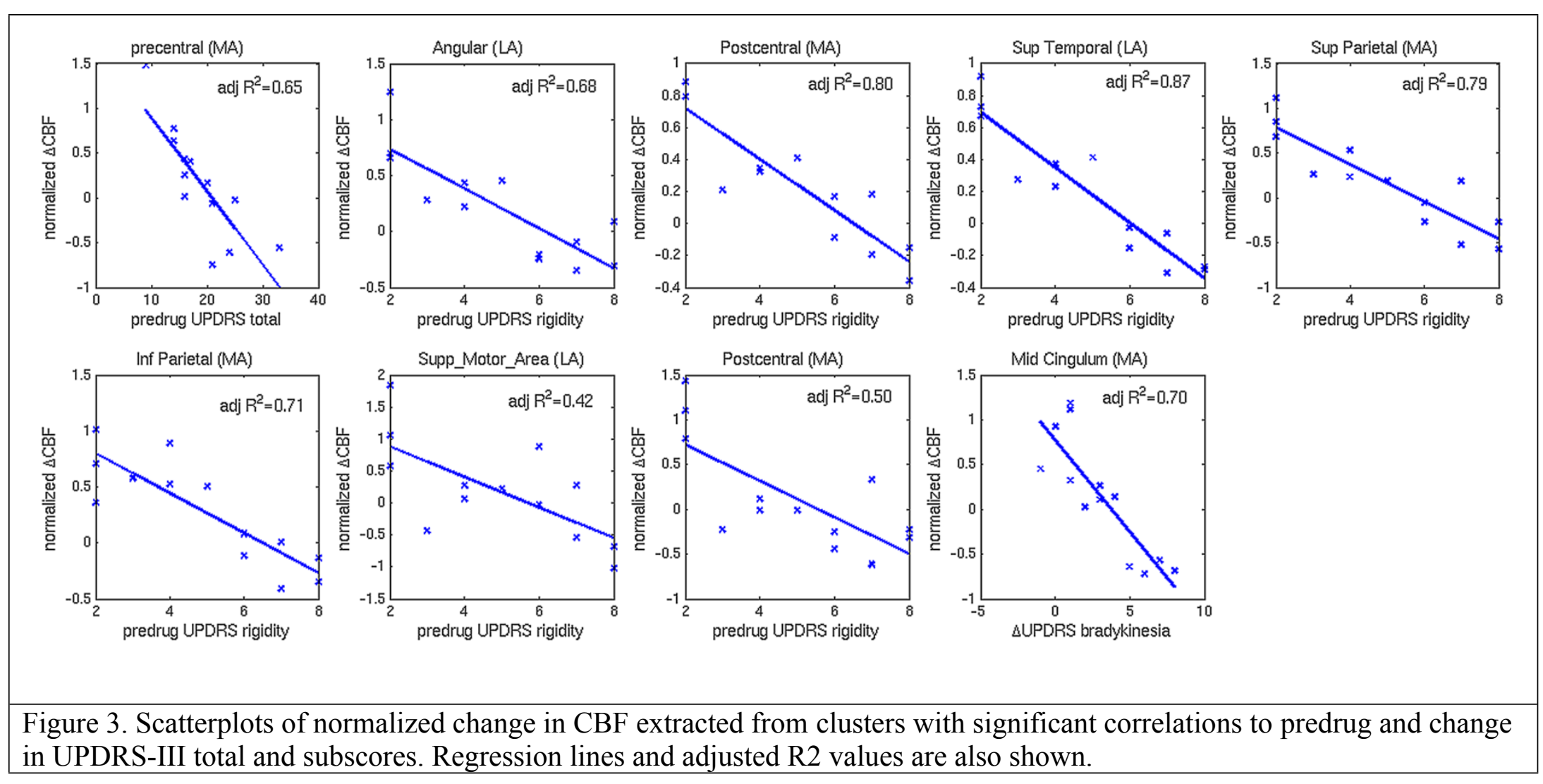


279

280

281

282

283

284

285

286

287

288

289

290

291

292

293

294

\section{Regression: Drug-induced CBF changes with LDE}

No significant correlation between drug-induced CBF changes and LDE were detected at the statistical threshold used.

\section{Discussion}

\section{Drug induced CBF change (pre vs. post)}

In this study, we used pCASL to measure CBF in PD patients before and after an oral dose of levodopa equivalent to $125 \%$ of their usual morning dosage of PD medications. The medication significantly improved motor functioning as expected; however, there was no significant change in global CBF. This finding is in agreement with several earlier ${ }^{15} \mathrm{O}-\mathrm{PET}$ studies investigating the effects of levodopa on CBF in both human PD patients and nonhuman primates(Hershey et al. 2003; Hershey et al. 2000; Hershey et al. 1998). The combination of the significant motor symptom benefits observed in our subjects and the lack of change in global CBF suggest that the levodopa dose administered in this study had minimal peripheral effects and had reached the intended target areas of the central nervous system, which were revealed by our voxel-based analysis.

Voxelwise paired t-test analysis between the proportionally scaled pre- and post-drug CBF maps revealed $\mathrm{CBF}$ increases after oral levodopa administration in several regions of the motor network including dorsal midbrain, (less affected) putamen/globus pallidus, and bilateral pre- and postcentral gyri. To further investigate the lack of bilateral results in the putamen/globus pallidi, we extracted putaminal CBF changes with hand-drawn posterior putamen masks. The left and right drug-induced putaminal CBF increases were $13 \%$ and $19 \%$ respectively, demonstrating 
301 bilateral putaminal response to levodopa. The lack of bilateral statistical significance is likely

302 due to a combination of lower subcortical CBF and significant partial volume effects for these

303 small structures. Our findings appear to contradict the negative findings from a similar ASL

304 study which used pulsed ASL to study the effects of a steady infusion of levodopa in 12 PD

305 patients(Stewart et al. 2014). The exact reason for this discrepancy is unknown. Since we did not

306 measure blood concentrations of levodopa in the current study, it is unclear whether the dosage

307 used is comparable between the two studies. Furthermore, the higher sensitivity of the TFCE

308 technique for statistical analysis used in the current study may account for the different findings.

309 In addition to affecting regions within the nigrostriatal pathway, levodopa also induced

310 CBF increases in the precuneus/cuneus, lingual, occipital and inferior parietal areas. While these

311 areas are not traditionally associated with movement control, prior studies have reported

312 perfusion deficits in similar regions for PD patients compared to healthy controls(Fernandez-

313 Seara et al. 2012; Melzer et al. 2011). Such findings support the idea of PD as a multifaceted

314 disease, as even regions extrinsic to the nigrostriatal network show significant involvement in PD.

315 Indeed, both occipital cortex(Koh et al. 2013; Tessitore et al. 2012b) and precuneus (Melzer et al.

316 2011; Tang \& Eidelberg 2010; Tang et al. 2010; Tessitore et al. 2012a) have shown alterations in

317 functional activation and structural integrity in Parkinson's disease. The response of these

318 regions to levodopa therefore may be mediated via their connections with other regions within

319 the nigrostriatal network. 
321

322

323

324

325

326

327

328

329

330

331

332

333

334

335

336

337

338

339

340

341

342

343

\section{Correlation between drug-induced CBF change (pre vs. post), change in UPDRS-III scores} and predrug UPDRS-III scores

In order to assess the regions of the brain that are associated with symptomatic improvement after levodopa administration, we correlated drug-induced CBF change to the change in UPDRS-III total and subscores. Only the change in bradykinesia subscore was significantly correlated with drug-induced CBF change, in a cluster located in the more affected mid-cingulate cortex, near the posterior end of Brodmann Area 24. The direction of the correlation was negative. Larger improvements are associated with smaller CBF changes. Both functional and diffusion tractography studies have demonstrated that the cingulate cortex can be segregated into functionally distinct regions(Vogt et al. 2003). A comparison between the coordinates of this cluster with previous studies appears to categorize this cluster in the cingulate motor area (CMA), which has extensive functional connections to sensory-motor areas including the primary motor cortex and spinal cord(Torta \& Cauda 2011) and is often activated during motor tasks(Beckmann et al. 2009). While the current finding appears in agreement with previous reports regarding the involvement of CMA in motor control, further research is necessary to elucidate why the CMA is associated with bradykinesia symptom improvement and not other parkinsonian symptoms.

We also performed a similar correlation analysis between $\mathrm{CBF}$ change and predrug UPDRS-III total and subscores to investigate whether disease severity affects the drug-induced CBF change. In this analysis, both total and rigidity UPDRS-III subscores were significantly correlated with CBF change. Specifically, the total UPDRS score was negatively correlated to CBF change in the more affected precentral gyrus. The rigidity subscore, on the other hand, revealed a widespread network of negative correlations, including bilateral postcentral gyri, 
344 superior motor area and parietal areas. The negative correlation indicates that more severe

345 clinical symptoms are associated with smaller CBF changes. This relationship is consistent with

346 the expectation that more severe clinical symptoms are likely associated with more disrupted

347 neurocircuitry, resulting in a more blunted drug response.

Although our results are in general agreement with findings in PD using other modalities,

it is important to emphasize that an assessment of regional CBF changes only partially addresses questions regarding underlying physiological processes. One ambiguity about CBF is that it is more tightly coupled to synaptic activity than spiking activity(Jueptner \& Weiller 1995;

Lauritzen 2001b), but it does not distinguish between excitatory or inhibitory input, making it difficult to determine downstream effects within a given network of regions. Also, an increase in CBF may not always represent an increase in local glucose metabolism or neural activity, as the typical tight coupling between $\mathrm{CBF}$ and metabolism may be disrupted in disease states. For example, concurrent $\mathrm{CBF}$ and glucose metabolism PET studies have shown in both rats and humans that levodopa induces a significant dissociation between CBF and glucose metabolism within the PD motor-related network, and that this dissociation is especially prominent in patients with levodopa-induced dyskinesia(Hirano et al. 2008; Ohlin et al. 2012). Future studies using multimodal techniques may help elucidate how functional hyperemia is affected. This study was limited by a small sample size, with no control for placebo effects, and not all participants had a robust response to levodopa. As we did not include healthy, agematched controls in this study, we are unable to determine whether the levodopa-induced CBF changes are specific to PD pathology. Correlation analysis between levodopa-induced CBF change and UPDRS motor scores suggest that the CBF response to levodopa may be different for patients on opposite ends of the severity spectrum. This remains to be demonstrated in a larger 
367 group of patients. Another limitation with the current study is the spatial resolution of the ASL 368 scan, which makes it difficult to resolve substructures in several of the brain regions implicated

369 in PD pathology, including the globus pallidus and substantia nigra, without partial volume

370 effects. This will be exacerbated when comparing patients to healthy controls where different

371 degrees of atrophy will also lead to variability in regional CBF. Higher resolution ASL

372 techniques, combined with partial volume correction, would provide a more accurate assessment

373 of local CBF changes. Finally, this study examined subjects with moderately advanced PD in the

374 OFF state (12 hours post last dose) and then the ON state. While this is an accepted method of

375 examining medications ON/ OFF effect, the OFF state does not reflect the true biological OFF

376 due to a long duration effect of PD medications(Hershey et al. 2003). Ideally, drug-naïve PD

377 patients should be imaged at baseline and followed longitudinally throughout their treatment for 378 a better understanding of the underlying mechanism.

\section{Conclusion}

We used ASL, a noninvasive MRI technique for measuring CBF, to explore the effects of an acute oral administration of levodopa on resting CBF in PD patients. Levodopa induced CBF increases in many regions of the brain, including major components of the nigrostriatal pathway, suggesting a combination of direct vascular and functional effects. Correlation analysis demonstrates a possible interaction between disease severity and CBF response to acute drug challenge. These results suggest the feasibility of ASL as a probe for the physiological effects of drugs in PD, which could not only further our understanding of current drug mechanisms, but 


\section{References}

Ashburner J, and Friston KJ. 2000. Voxel-based morphometry--the methods. Neuroimage 11:805-821. 10.1006/nimg.2000.0582

Beckmann M, Johansen-Berg H, and Rushworth MF. 2009. Connectivity-based parcellation of human cingulate cortex and its relation to functional specialization. The Journal of neuroscience : the official journal of the Society for Neuroscience 29:1175-1190. 10.1523/JNEUROSCI.3328-08.2009

Buhmann C, Glauche V, Sturenburg HJ, Oechsner M, Weiller C, and Buchel C. 2003. Pharmacologically modulated $\mathrm{fMRI--cortical} \mathrm{responsiveness} \mathrm{to} \mathrm{levodopa} \mathrm{in} \mathrm{drug-naive}$ hemiparkinsonian patients. Brain : a journal of neurology 126:451-461.

Chen Y, Wan HI, O'Reardon JP, Wang DJ, Wang Z, Korczykowski M, and Detre JA. 2011. Quantification of cerebral blood flow as biomarker of drug effect: arterial spin labeling phMRI after a single dose of oral citalopram. Clin Pharmacol Ther 89:251-258. clpt2010296 [pii]. 10.1038/clpt.2010.296

Chen Y, Wang D, and Detre JA. 2010. Test-Retest Reliability of Arterial Spin Labeling with Common Labeling Strategies. Journal of magnetic resonance imaging.

Dai W, Garcia D, de Bazelaire C, and Alsop DC. 2008. Continuous flow-driven inversion for arterial spin labeling using pulsed radio frequency and gradient fields. Magn Reson Med 60:1488-1497. 10.1002/mrm.21790

Detre JA, Leigh JS, Williams DS, and Koretsky AP. 1992. Perfusion imaging. Magn Reson Med 23:37-45.

Eidelberg D. 2009. Metabolic brain networks in neurodegenerative disorders: a functional imaging approach. Trends in neurosciences 32:548-557. 10.1016/j.tins.2009.06.003

Eidelberg D, Moeller JR, Dhawan V, Sidtis JJ, Ginos JZ, Strother SC, Cedarbaum J, Greene P, Fahn S, and Rottenberg DA. 1990. The metabolic anatomy of Parkinson's disease: complementary [18F]fluorodeoxyglucose and [18F]fluorodopa positron emission tomographic studies. Movement disorders : official journal of the Movement Disorder Society 5:203-213. 10.1002/mds.870050304

Fahn S ER, Members of the UPDRS Development Committee. 1987. The Unified Parkinson's DIsease Rating Scale. Recent Developments in Parkinson's Disease. Florhan Park, NJ: Raven Press, 153-163, 293-304.

Feigin A, Ghilardi MF, Fukuda M, Mentis MJ, Dhawan V, Barnes A, Ghez CP, and Eidelberg D. 2002. Effects of levodopa infusion on motor activation responses in Parkinson's disease. Neurology 59:220-226. 
423

424

425

426

427

428

429

430

431

432

433

434

435

436

437

438

439

440

441

442

443

444

445

446

447

448

449

450

451

452

453

454

455

456

457

458

Fernandez-Seara MA, Mengual E, Vidorreta M, Aznarez-Sanado M, Loayza FR, Villagra F, Irigoyen J, and Pastor MA. 2012. Cortical hypoperfusion in Parkinson's disease assessed using arterial spin labeled perfusion MRI. NeuroImage 59:2743-2750. 10.1016/j.neuroimage.2011.10.033

Gevers S, Majoie CB, van den Tweel XW, Lavini C, and Nederveen AJ. 2009. Acquisition time and reproducibility of continuous arterial spin-labeling perfusion imaging at 3T. AJNR Am J Neuroradiol 30:968-971. ajnr.A1454 [pii]

10.3174/ajnr.A1454

Guttman M, Yong VW, Kim SU, Calne DB, Martin WR, Adam MJ, and Ruth TJ. 1988. Asymptomatic striatal dopamine depletion: PET scans in unilateral MPTP monkeys. Synapse 2:469-473. 10.1002/syn.890020502

Hermes M, Hagemann D, Britz P, Lieser S, Rock J, Naumann E, and Walter C. 2007. Reproducibility of continuous arterial spin labeling perfusion MRI after 7 weeks. MAGMA 20:103-115. 10.1007/s10334-007-0073-3

Hershey T, Black KJ, Carl JL, McGee-Minnich L, Snyder AZ, and Perlmutter JS. 2003. Long term treatment and disease severity change brain responses to levodopa in Parkinson's disease. Journal of neurology, neurosurgery, and psychiatry 74:844-851.

Hershey T, Black KJ, Carl JL, and Perlmutter JS. 2000. Dopa-induced blood flow responses in nonhuman primates. Experimental neurology 166:342-349. 10.1006/exnr.2000.7522

Hershey T, Black KJ, Stambuk MK, Carl JL, McGee-Minnich LA, and Perlmutter JS. 1998. Altered thalamic response to levodopa in Parkinson's patients with dopa-induced dyskinesias. Proceedings of the National Academy of Sciences of the United States of America 95:12016-12021.

Hirano S, Asanuma K, Ma Y, Tang C, Feigin A, Dhawan V, Carbon M, and Eidelberg D. 2008. Dissociation of metabolic and neurovascular responses to levodopa in the treatment of Parkinson's disease. The Journal of neuroscience : the official journal of the Society for Neuroscience 28:4201-4209. 10.1523/JNEUROSCI.0582-08.2008

Hornykiewicz O. 2010. A brief history of levodopa. Journal of neurology 257:S249-252. 10.1007/s00415-010-5741-y

Huang WS, Lin SZ, Lin JC, Wey SP, Ting G, and Liu RS. 2001. Evaluation of early-stage Parkinson's disease with 99mTc-TRODAT-1 imaging. Journal of nuclear medicine : official publication, Society of Nuclear Medicine 42:1303-1308.

Jueptner M, and Weiller C. 1995. Review: does measurement of regional cerebral blood flow reflect synaptic activity? Implications for PET and fMRI. Neuroimage 2:148-156.

Kamagata K, Motoi Y, Hori M, Suzuki M, Nakanishi A, Shimoji K, Kyougoku S, Kuwatsuru R, Sasai K, Abe O, Mizuno Y, Aoki S, and Hattori N. 2011. Posterior hypoperfusion in 
459

460

461

462

463

464

465

466

467

468

469

470

471

472

473

474

475

476

477

478

479

480

481

482

483

484

485

486

487

488

489

490

491

492

493

494

Parkinson's disease with and without dementia measured with arterial spin labeling MRI. Journal of magnetic resonance imaging : JMRI 33:803-807. 10.1002/jmri.22515

Kobari M, Fukuuchi Y, Shinohara T, Obara K, and Nogawa S. 1995. Levodopa-induced local cerebral blood flow changes in Parkinson's disease and related disorders. Journal of the neurological sciences 128:212-218.

Koh SB, Suh SI, Kim SH, and Kim JH. 2013. Stereopsis and extrastriate cortical atrophy in Parkinson's disease: a voxel-based morphometric study. Neuroreport 24:229-232.

Kriegeskorte N, Lindquist MA, Nichols TE, Poldrack RA, and Vul E. 2010. Everything you never wanted to know about circular analysis, but were afraid to ask. J Cereb Blood Flow Metab 30:1551-1557. 10.1038/jcbfm.2010.86

Lauritzen M. 2001a. Relationship of spikes, synaptic activity, and local changes of cerebral blood flow. Journal of cerebral blood flow and metabolism : official journal of the International Society of Cerebral Blood Flow and Metabolism 21:1367-1383. 10.1097/00004647-200112000-00001

Lauritzen M. 2001b. Relationship of spikes, synaptic activity, and local changes of cerebral blood flow. J Cereb Blood Flow Metab 21:1367-1383. 10.1097/00004647-20011200000001

Ma Y, Tang C, Moeller JR, and Eidelberg D. 2009. Abnormal regional brain function in Parkinson's disease: truth or fiction? NeuroImage 45:260-266. 10.1016/j.neuroimage.2008.09.052

Melzer TR, Watts R, MacAskill MR, Pearson JF, Rueger S, Pitcher TL, Livingston L, Graham C, Keenan R, Shankaranarayanan A, Alsop DC, Dalrymple-Alford JC, and Anderson TJ. 2011. Arterial spin labelling reveals an abnormal cerebral perfusion pattern in Parkinson's disease. Brain : a journal of neurology 134:845-855.

Morrish PK, Sawle GV, and Brooks DJ. 1995. Clinical and [18F] dopa PET findings in early Parkinson's disease. Journal of neurology, neurosurgery, and psychiatry 59:597-600.

Nichols TE, and Holmes AP. 2002. Nonparametric permutation tests for functional neuroimaging: a primer with examples. Hum Brain Mapp 15:1-25.

Ohlin KE, Sebastianutto I, Adkins CE, Lundblad C, Lockman PR, and Cenci MA. 2012. Impact of L-DOPA treatment on regional cerebral blood flow and metabolism in the basal ganglia in a rat model of Parkinson's disease. NeuroImage 61:228-239. 10.1016/j.neuroimage.2012.02.066

Paschali A, Messinis L, Kargiotis O, Lakiotis V, Kefalopoulou Z, Constantoyannis C, Papathanasopoulos P, and Vassilakos P. 2010. SPECT neuroimaging and neuropsychological functions in different stages of Parkinson's disease. European journal of nuclear medicine and molecular imaging 37:1128-1140. 10.1007/s00259-010-1381-9 
495

496

497

498

499

500

501

502

503

504

505

506

507

508

509

510

511

512

513

514

515

516

517

518

519

520

521

522

523

524

525

526

527

528

529

530

531

Prakash BD, Sitoh YY, Tan LC, and Au WL. 2012. Asymmetrical diffusion tensor imaging indices of the rostral substantia nigra in Parkinson's disease. Parkinsonism \& related disorders 18:1029-1033. 10.1016/j.parkreldis.2012.05.021

Rinne JO, Laihinen A, Rinne UK, Nagren K, Bergman J, and Ruotsalainen U. 1993. PET study on striatal dopamine D2 receptor changes during the progression of early Parkinson's disease. Movement disorders : official journal of the Movement Disorder Society 8:134138. $10.1002 / \mathrm{mds} .870080203$

Schuff N, Wu IW, Buckley S, Foster ED, Coffey CS, Gitelman DR, Mendick S, Seibyl J, Simuni T, Zhang Y, Jankovic J, Hunter C, Tanner CM, Rees L, Factor S, Berg D, Wurster I, Gauss K, Sprenger F, Seppi K, Poewe W, Mollenhauer B, Knake S, Mari Z, McCoy A, Ranola M, and Marek K. 2015. Diffusion imaging of nigral alterations in early Parkinson's disease with dopaminergic deficits. Movement disorders : official journal of the Movement Disorder Society. 10.1002/mds.26325

Smith SM, and Nichols TE. 2009. Threshold-free cluster enhancement: addressing problems of smoothing, threshold dependence and localisation in cluster inference. Neuroimage 44:83-98. 10.1016/j.neuroimage.2008.03.061

Song IU, Yoo I, Chung YA, and Jeong J. 2015. The value of brain perfusion SPECT for differentiation between mildly symptomatic idiopathic Parkinson's disease and the Parkinson variant of multiple system atrophy. Nuclear medicine communications. 10.1097/MNM.0000000000000354

Stewart SB, Koller JM, Campbell MC, and Black KJ. 2014. Arterial spin labeling versus BOLD in direct challenge and drug-task interaction pharmacological fMRI. PeerJ 2:e687. 10.7717 peerj.687

Stoessl AJ. 2012. Neuroimaging in Parkinson's disease: from pathology to diagnosis. Parkinsonism \& related disorders 18 Suppl 1:S55-59. 10.1016/S1353-8020(11)70019-0

Tang CC, and Eidelberg D. 2010. Abnormal metabolic brain networks in Parkinson's disease from blackboard to bedside. Progress in brain research 184:161-176. 10.1016/S00796123(10)84008-7

Tang CC, Poston KL, Dhawan V, and Eidelberg D. 2010. Abnormalities in metabolic network activity precede the onset of motor symptoms in Parkinson's disease. Journal of Neuroscience 30:1049-1056.

Tessitore A, Amboni M, Cirillo G, Corbo D, Picillo M, Russo A, Vitale C, Santangelo G, Erro R, Cirillo M, Esposito F, Barone P, and Tedeschi G. 2012a. Regional gray matter atrophy in patients with Parkinson disease and freezing of gait. Ajnr: American Journal of Neuroradiology 33:1804-1809.

Tessitore A, Amboni M, Esposito F, Russo A, Picillo M, Marcuccio L, Pellecchia MT, Vitale C, Cirillo M, Tedeschi G, and Barone P. 2012b. Resting-state brain connectivity in patients 
with Parkinson's disease and freezing of gait. Parkinsonism \& related disorders 18:781787.

Teune LK, Renken RJ, de Jong BM, Willemsen AT, van Osch MJ, Roerdink JB, Dierckx RA, and Leenders KL. 2014. Parkinson's disease-related perfusion and glucose metabolic brain patterns identified with PCASL-MRI and FDG-PET imaging. NeuroImage Clinical 5:240-244. 10.1016/j.nicl.2014.06.007

Tomlinson CL, Stowe R, Patel S, Rick C, Gray R, and Clarke CE. 2010. Systematic review of levodopa dose equivalency reporting in Parkinson's disease. Movement disorders : official journal of the Movement Disorder Society 25:2649-2653. 10.1002/mds.23429

Torta DM, and Cauda F. 2011. Different functions in the cingulate cortex, a meta-analytic connectivity modeling study. NeuroImage 56:2157-2172. 10.1016/j.neuroimage.2011.03.066

Tzourio-Mazoyer N, Landeau B, Papathanassiou D, Crivello F, Etard O, Delcroix N, Mazoyer B, and Joliot M. 2002. Automated anatomical labeling of activations in SPM using a macroscopic anatomical parcellation of the MNI MRI single-subject brain. Neuroimage 15:273-289. 10.1006/nimg.2001.0978

van Osch MJ, Teeuwisse WM, van Walderveen MA, Hendrikse J, Kies DA, and van Buchem MA. 2009. Can arterial spin labeling detect white matter perfusion signal? Magnetic resonance in medicine 62:165-173. 10.1002/mrm.22002

Vogt BA, Berger GR, and Derbyshire SW. 2003. Structural and functional dichotomy of human midcingulate cortex. The European journal of neuroscience 18:3134-3144.

Wang DJ, Chen Y, Fernandez-Seara MA, and Detre JA. 2011. Potentials and challenges for arterial spin labeling in pharmacological magnetic resonance imaging. The Journal of pharmacology and experimental therapeutics 337:359-366. 10.1124/jpet.110.172577

Wang Z, Aguirre GK, Rao H, Wang J, Fernandez-Seara MA, Childress AR, and Detre JA. 2008. Empirical optimization of ASL data analysis using an ASL data processing toolbox: ASLtbx. Magn Reson Imaging 26:261-269. 10.1016/j.mri.2007.07.003

Yang J, Sadler TR, Givrad TK, Maarek JM, and Holschneider DP. 2007. Changes in brain functional activation during resting and locomotor states after unilateral nigrostriatal damage in rats. NeuroImage 36:755-773. 10.1016/j.neuroimage.2007.03.010 


\section{Figure Captions}

566 Figure 1. Results of voxel-wise paired t-test between pre- and post-drug CBF maps thresholded

567 at $\mathrm{p}<0.05$, FWE corrected for multiple comparisons, overlaid onto a representative subject's

568 spatially normalized anatomical image. Images are oriented so that the "more affected" side of

569 the brain is on the left side of the image. Orange represents CBF increases. No CBF decreases

570 were detected at the statistical threshold used.

571 Figure 2. Clusters with significant correlation between a) change in CBF and change in

572 bradykinesia subscore, b) change in $\mathrm{CBF}$ and predrug rigidity subscore and c) change in $\mathrm{CBF}$

573 and predrug total UPDRS-III score. Blue represents negative correlations, where larger

574 improvements were associated with smaller CBF changes. No positive correlations were detected

575 at the statistical threshold used.

576 Figure 3. Scatterplots of normalized change in CBF extracted from clusters with significant

577 correlations to predrug and change in UPDRS-III total and subscores. Regression lines and

578 adjusted R2 values are also shown. 
1

Results of voxel-wise paired t-test between pre- and post-drug CBF maps

Figure 1. Results of voxel-wise paired t-test between pre- and post-drug CBF maps thresholded at $p<0.05$, FWE corrected for multiple comparisons, overlaid onto a representative subject's spatially normalized anatomical image. Images are oriented so that the "more affected" side of the brain is on the left side of the image. Orange represents CBF increases. No CBF decreases were detected at the statistical threshold used.

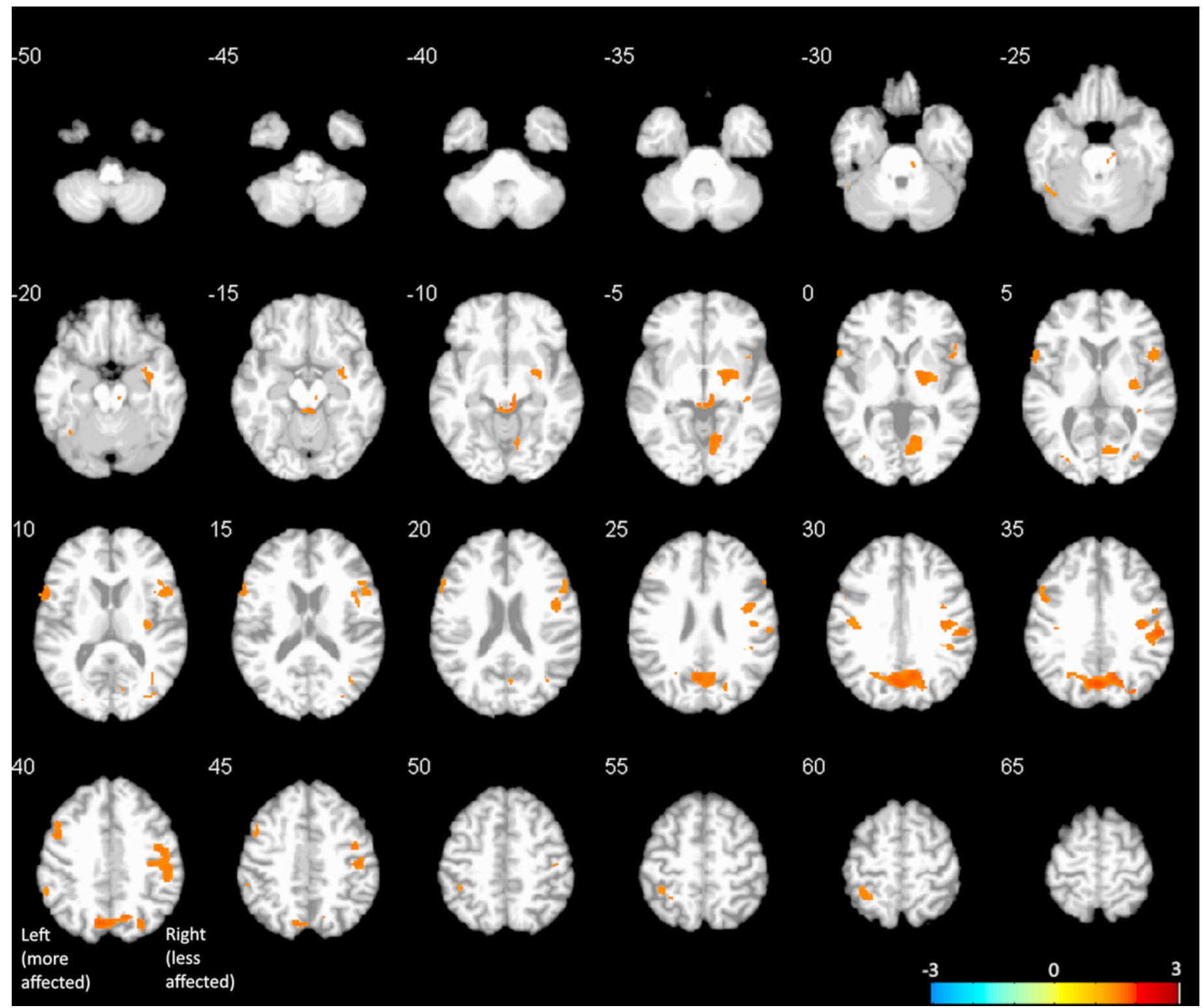


2

Clusters with significant correlation between normalized changes in CBF and UPDRS-III subscores

Figure 2. Clusters with significant correlation between a) change in CBF and change in bradykinesia subscore, b) change in CBF and predrug rigidity subscore and c) change in CBF and predrug total UPDRS-III score. Blue represents negative correlations, where larger improvements were associated with smaller CBF changes. No positive correlations were detected at the statistical threshold used. 


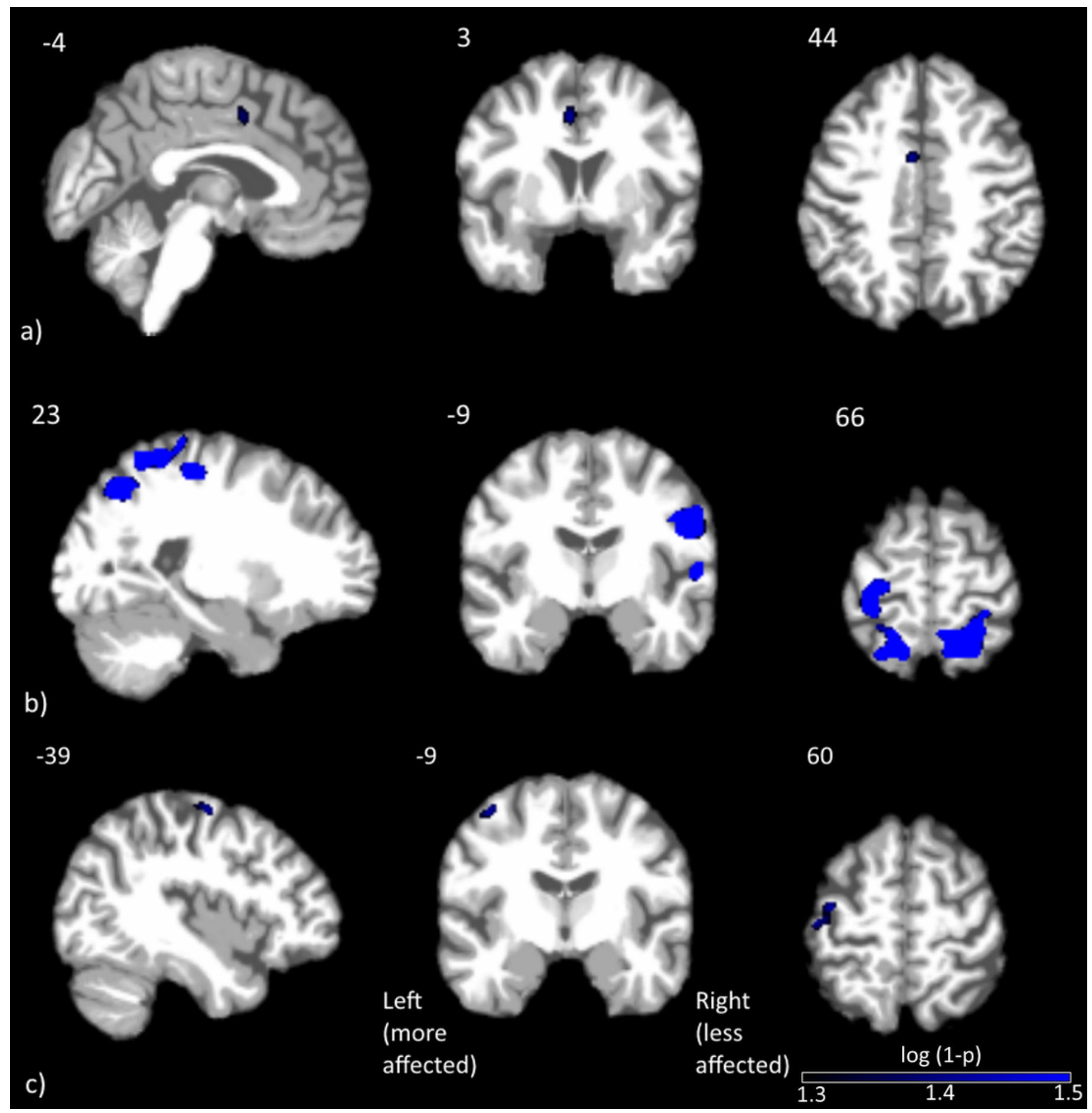


3

Scatterplots of normalized changes in CBF and UPDRS-III subscores

Figure 3. Scatterplots of normalized change in CBF extracted from clusters with significant correlations to predrug and change in UPDRS-III total and subscores. Regression lines and adjusted $R^{2}$ values are also shown.
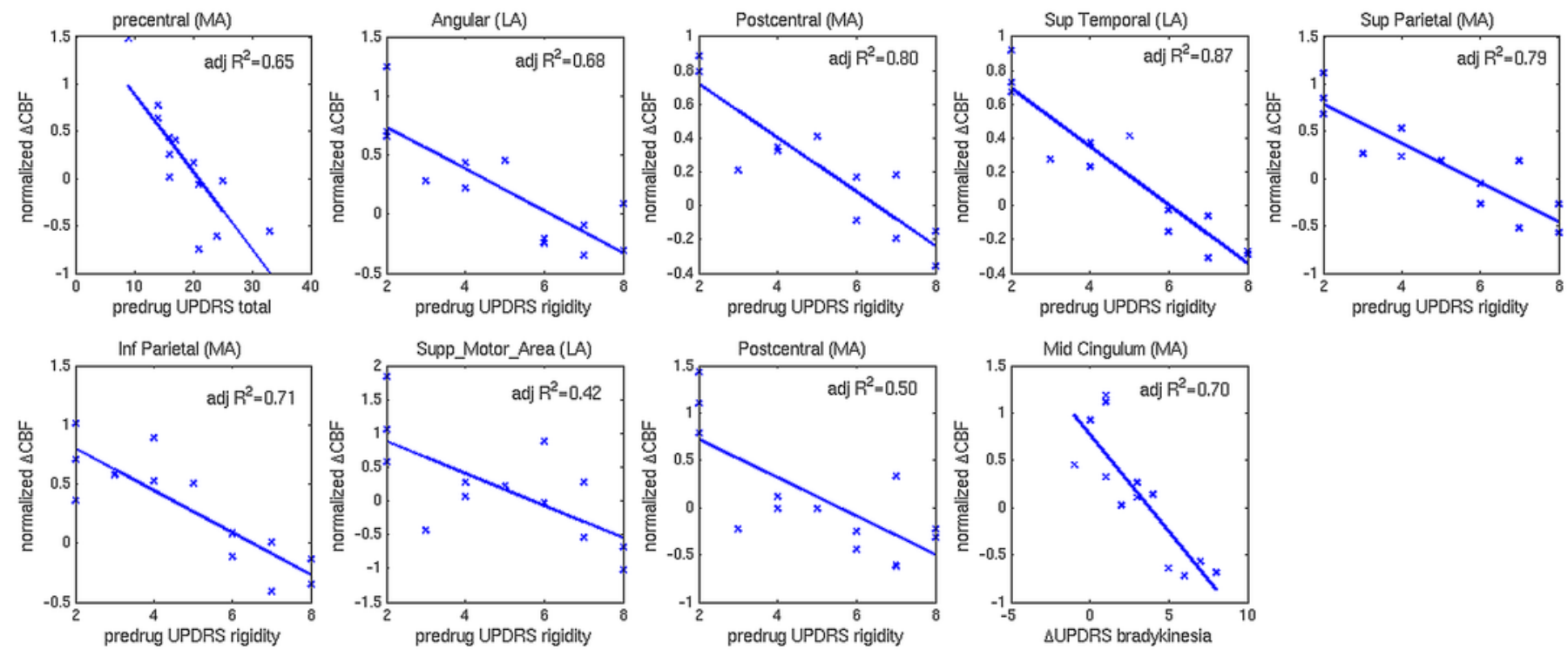


\section{Table $\mathbf{1}$ (on next page)}

Demographics, UPDRS-III ratings and Levodopa-equivalence (LDE) in mg.

Demographics, UPDRS-III ratings and Levodopa-equivalence (LDE) in mg. 
1 Table 1. Demographics, UPDRS-III ratings and Levodopa-equivalence (LDE) in mg.

\begin{tabular}{lr}
\hline \multicolumn{2}{c}{ Demographics $(\mathbf{n}=\mathbf{1 3})$} \\
\hline Age, years (mean \pm s.d.) & $65 \pm 7$ \\
\hline Female:male & $3: 10$ \\
Duration of disease (years) & $8 \pm 6$ \\
LDE*, mg (mean \pm s.d.) & $270 \pm 132$ \\
L vs R dominant symptoms & $5: 8$
\end{tabular}

\begin{tabular}{lrrr}
\hline \multicolumn{4}{c}{ Drug Response (n=13) } \\
\hline & \multicolumn{1}{c}{ pre } & \multicolumn{1}{c}{ post } & \%improvement \\
\hline UPDRS-III total(mean \pm s.d.) $^{\prime} 19 \pm 6$ & $9 \pm 6$ & $52.0 \pm 28.3$ \\
Bradykinesia subscore $^{\dagger}$ & $7 \pm 4$ & $4 \pm 4$ & $49.1 \pm 38.8$ \\
Tremor subscore $^{\text {Rigidity subscore }}$ & $3 \pm 2$ & $1 \pm 2$ & $58.3 \pm 43.3$ \\
Posture subscore & $5 \pm 2$ & $3 \pm 2$ & $46.8 \pm 34.5$ \\
\hline
\end{tabular}

2

$3 * \mathrm{LDE}=$ levodopa equivalents based on Tomlinson et al (2010) for the morning dose of

4 dopaminergic medications.

5 †See text for the formulas for the calculation of the UPDRS subscores.

6 


\section{Table 2 (on next page)}

List of clusters with significant CBF increases after levodopa administration.

List of clusters with significant CBF increases after levodopa administration. MNI coordinates of the peak voxel are given in $\mathrm{mm}$, as well as the number of voxels in each cluster and FWE p-value. Mean percent CBF changes were calculated from a $5 \mathrm{~mm}$ radius sphere centered at the peak voxel. 
1 Table 2. List of clusters with significant CBF increases after levodopa administration. MNI 2 coordinates of the peak voxel are given in $\mathrm{mm}$, as well as the number of voxels in each cluster 3 and FWE p-value. Mean percent CBF changes were calculated from a $5 \mathrm{~mm}$ radius sphere 4 centered at the peak voxel.

\begin{tabular}{|c|c|c|c|c|}
\hline $\mathbf{x}, \mathbf{y}, \mathbf{z}, \mathbf{m m}$ & $\begin{array}{c}\text { No. } \\
\text { voxels }\end{array}$ & $\begin{array}{c}\text { FWE } \\
\text { p-value }\end{array}$ & label & $\begin{array}{l}\text { \% CBF } \\
\text { change }\end{array}$ \\
\hline $0-7033$ & 2695 & 0.016 & Cuneus, precuneus & 33.4 \\
\hline $56-2434$ & 3046 & 0.020 & $\begin{array}{c}\text { SupraMarginal (LA), } \\
\text { Frontal_Inf_Oper (LA), } \\
\text { Postcentral (LA), Precentral (LA) }\end{array}$ & 26.9 \\
\hline $4-33-9$ & 544 & 0.027 & midbrain & 73.6 \\
\hline $20-6-3$ & 1150 & 0.029 & Pallidum (LA), putamen (LA) & 44.0 \\
\hline-60119 & 461 & 0.030 & Frontal_Inf_Oper (MA) & 50.6 \\
\hline $10-64-5$ & 863 & 0.030 & Lingual (LA) & 33.0 \\
\hline-48839 & 428 & 0.031 & Precentral (MA) & 31.0 \\
\hline$-34-4660$ & 314 & 0.043 & Parietal_Inf (MA) & 61.1 \\
\hline $34-799$ & 108 & 0.043 & Occipital_Mid (LA) & 40.9 \\
\hline$-40-1830$ & 146 & 0.044 & Postcentral (MA) & 47.1 \\
\hline $38-6915$ & 77 & 0.044 & White matter & 47.8 \\
\hline$-40-57-23$ & 108 & 0.045 & Cerebelum_6(LA) & 36.2 \\
\hline $40-27-6$ & 57 & 0.045 & White matter & 32.1 \\
\hline$-27-857$ & 59 & 0.045 & Occipital_Mid (MA) & 47.3 \\
\hline$-58-4539$ & 77 & 0.047 & Parietal Inf (MA) & 30.1 \\
\hline$-8-28-14$ & 2 & 0.048 & midbrain & 62.5 \\
\hline $38-396$ & 26 & 0.049 & White matter & 85.8 \\
\hline $\begin{array}{llll}-50 & 30 & 25\end{array}$ & 5 & 0.050 & Frontal_Inf_Tri (MA) & 41.8 \\
\hline
\end{tabular}

5

$6 \quad \mathrm{MA}=$ more affected; LA=less affected.

7 Note that left cerebellum was designated as less affected because motor fibers decussate in the 8 medulla oblongata. 


\section{Table 3(on next page)}

List of clusters with significant correlations between normalized $\square$ CBF and predrug or change in UPDRS scores.

List of clusters with significant correlations between normalized $\square$ CBF and predrug or change in UPDRS scores. MNI coordinates of the peak voxel are given in $\mathrm{mm}$, as well as the number of voxels in each cluster and FWE p-value. 
1 Table 3. List of clusters with significant correlations between normalized $\triangle \mathrm{CBF}$ and predrug or 2 change in UPDRS scores. MNI coordinates of the peak voxel are given in $\mathrm{mm}$, as well as the 3 number of voxels in each cluster and FWE p-value.

\begin{tabular}{ccccc}
\hline & $\mathbf{x , y}, \mathbf{z}, \mathbf{m m}$ & $\begin{array}{c}\text { No. } \\
\text { voxels }\end{array}$ & $\begin{array}{c}\text { FWE } \\
\mathbf{p}-\mathbf{v a l u e}\end{array}$ & label \\
\hline $\begin{array}{c}\text { BUPDRS } \\
\begin{array}{c}\text { Bradykinesia } \\
\text { (negative) }\end{array}\end{array}$ & $-4,3,43$ & 88 & 0.043 & Cingulum_Mid (MA) \\
$\begin{array}{c}\text { Pre-drug UPDRS } \\
\text { Rigidity (negative) }\end{array}$ & $38,-57,54$ & 1997 & 0.009 & Angular(LA) \\
& $48,-12,30$ & 987 & 0.010 & Postcentral (LA) \\
& $58,-27,12$ & 4617 & 0.045 & Temporal_Sup (LA) \\
& $-27,-60,61$ & 2547 & 0.019 & Parietal_Sup (MA) \\
& $-46,-48,52$ & 457 & 0.032 & Parietal_Inf (MA) \\
& $2,-21,72$ & 6 & 0.044 & Supp_Motor_Area (LA) \\
Total (negative) & $-34,-24,49$ & 106 & 0.045 & Postcentral (MA) \\
\hline
\end{tabular}

$4 \quad \mathrm{MA}=$ more affected; LA=less affected. 